\title{
The impact of sperm-expressed transcription factors on fate-mapping models
}

\author{
Xin Yuan ${ }^{1, *}$, Jianlin $\mathrm{Du}^{2,{ }^{*}}$, Qin Qin ${ }^{2}$, Xiaoqun $\mathrm{Li}^{2}$, Songbai Deng ${ }^{2}$, Yunqing Chen ${ }^{2}$, \\ Ling Zhang ${ }^{1}$ and Qiang She ${ }^{2}$ \\ Departments of ${ }^{1}$ Nephrology and ${ }^{2}$ Cardiology, Second Affiliated Hospital of Chongqing Medical University, \\ Chongqing 400010, China \\ Correspondence should be addressed to Q She; Email: qshe98@hotmail.com \\ ${ }^{*}(\mathrm{X}$ Yuan and J L Du contributed equally to this work)
}

\begin{abstract}
Genetic lineage tracing has been used extensively in developmental biology. Many transcription factors expressed in sperm may induce Cre-mediated loxP recombination during early zygote development. In this study, we investigated the effect of sperm-expressed Cre on cell type-specific Cre-mediated loxP recombination in fate-mapping models of $\mathrm{Tbx}^{+} \mathrm{P}^{+}$progenitor cells. We found the recombination frequency in a reverse mating (RM) lineage was inconsistent with a normal Mendelian distribution. However, the recombination frequency in a positive mating (PM) lineage agreed with a Mendelian distribution. In the PM lineage, LacZ and EYFP were expressed in specific locations, such as the limb buds, heart, and hair follicles. Therefore, the reporter genes accurately and reliably traced cell differentiation in the PM lineage. In contrast, EYFP and LacZ were expressed throughout the embryo in the RM lineage. Thus, the reporter genes did not trace cell differentiation specifically in the RM lineage. Furthermore, Tbx18 mRNA and protein were expressed in the testicles of male mice, but almost no Tbx18 expression was detected in the ovaries of female mice. Similarly, reporter genes and Tbx18 were coexpressed in the seminiferous tubules and sperm cells of testicles. These results revealed that Cre-loxP-mediated prerecombination in zygotes is due to Tbx18 expressed in testicle sperm cells when Cre is transmitted paternally. Our results indicate that Cre-mediated specific recombination in fate-mapping models of sperm-expressed genes may be influenced by the paternal origin of Cre. Therefore, a careful experimental design is critical when using the Cre-loxP system to trace spatial, temporal or tissue-specific fates. Reproduction (2015) $150323-330$
\end{abstract}

\section{Introduction}

Lineage tracing is an essential tool for identifying the properties of all of the progeny of stem and progenitor cells in adult and embryonic tissues (Kretzschmar \& Watt 2012). Genetic lineage tracing, which marks cells by genetic recombination, has been used extensively in developmental biology to explore which stem/progenitor cells in early embryos generate a specific structure, to identify their derivatives and to investigate the mechanism of differentiation (Laforest et al. 2014). The Cre-loxP system is most commonly used for genetic lineage tracing in mice (Kretzschmar \& Watt 2012). Genetic lineage tracing is one of the most reliable means of monitoring the cell fate of cardiac progenitor cells in vivo and in vitro. Cre reporter mice, such as Rosa26 $6^{\text {LacZ }}$ and Rosa26 $6^{\text {EYFP }}$, are useful for cell fate-mapping experiments and for monitoring Cre expression (Soriano 1999, Srinivas et al. 2001). Many transcription factors are expressed in sperm and eggs, and they may induce Cre-mediated excision of the loxP-flanked transcriptional 'stop' sequence during early zygote and embryonic stem cell development. Previous studies revealed that Tbx18 is important in the normal development of many different tissues and organs, including the heart, kidney, ureter, bladder, heart, limb, somite, hair follicle, and cochlea, and during mouse embryogenesis (Bussen et al. 2004, Cai et al. 2008, Trowe et al. 2008, Wehn \& Chapman 2010, Grisanti et al. 2013, Xu et al. 2014). Tbx $18^{+}$progenitor cells differentiate into pacemaker cells, smooth muscle cells, and fibroblasts in the heart (Cai et al. 2008, Wiese et al. 2009, Liang et al. 2013, Wu et al. 2013). Similarly, Bohnenpoll et al. have shown that $\mathrm{Tb} 18^{+}$cells are a pool of multipotent progenitor cells that contribute to stromal cells, mesangial cells, vascular smooth muscle cells within the kidney, and smooth muscle cells within the ureter and bladder (Bohnenpoll et al. 2013, Xu et al. 2014, Yan et al. 2014). Therefore, Tbx18 has been identified as a marker of multipotent progenitor cells. To monitor the fate of $\mathrm{Tbx} 18^{+}$progenitor cells in vivo and in vitro, Tbx18Cre/R26 $6^{\text {LacZ }}$ and Tbx18Cre/R26 $6^{\text {EYFP }}$ fate-mapping mice have been produced by crossing 
Tbx18Cre mice with Rosa26 $6^{\text {lacz }}$ and Rosa26 $6^{\text {EYFP }}$ mice, respectively. Tbx18Cre knock-in mouse lines have been established by inserting a Cre-PGK-neo cassette into exon 1 of Tbx18 (Cai et al. 2008, Christoffels et al. 2009, Wiese et al. 2009, Bohnenpoll et al. 2013) or introducing a BAC-ICre ampicillin cassette into exon 1 of Tbx18 (Wang et al. 2009). However, these three Tbx18cre mice show partially different results in the heart and urinary system. The heart tissue revealed that Tbx18-expressing epicardial cells contribute to cardiomyocytes in the interventricular septum of Tbx18cre mice (Cai et al. 2008). In contrast, the genetic lineage study by Bohnenpoll et al. revealed that Tbx18-Cre cell lineage tracing with the PGK-neo cassette targeting vector does not contribute unequivocally to cardiomyocytes in the interventricular septum in vivo (Christoffels et al. 2009). Therefore, it is important to determine the influence of Cre-mediated recombination on reporter gene expression (e.g., LacZ and EYFP) in germline cells. However, it is not clear whether Cre recombinase expression is influenced by the distinctive spatiotemporal expression patterns of these reporter genes, as the origins of Cre have differed in these mouse models (paternal or maternal lineage), and Cre is activated by a Tbx18 promoter in the sperm or eggs of genetic lineage tracing mice (Cai et al. 2008, Christoffels et al. 2009, Smith 2011, Lee et al. 2013). Thus, this study assessed the effects of Cre-mediated recombination in male germ cells on reporter gene expression used for lineage tracing. We conducted genotyping analyses by PCR, and we performed X-gal or immunofluorescence staining for $\mathrm{LacZ}$ or EYFP expression to identify the Mendelian law and the specificity of the positioning of reporter gene expression in fate-mapping mice of $\mathrm{Tbx}^{+} 8^{+}$progenitor cells. Based on our results, we reveal that Cre-mediated site-specific recombination in fate-mapping models of a spermexpressed transcription factor is influenced by the paternal origin of Cre.

\section{Materials and methods}

\section{Mice}

Tbx18Cre mice were genotyped by PCR as previously described (Cai et al. 2008). The Rosa $26^{\mathrm{EYFP}}$ and Rosa $26 \mathrm{R}^{\text {lacZ }}$ reporter mice (Soriano 1999, Srinivas et al. 2001) were purchased from the Jackson Laboratory (Bar Harbor, ME, USA). The fate-mapping models were established by crossing female Tbx18Cre mice with male Rosa26R lacZ mice and female Tbx18Cre mice with male Rosa $26 R^{\text {EYFP }}$ mice. These matings were defined by having a maternal Cre origin (positive mating (PM)). Models were also established by crossing male Tbx18Cre mice with female Rosa26R $\mathrm{R}^{\text {lac } Z}$ mice and male Tbx18Cre mice with female Rosa26R ${ }^{\text {EYFP }}$ mice. These matings were defined by a paternal Cre (reverse mating (RM)). Tbx18Cre, Rosa26 $6^{\mathrm{EYFP}}$, and Rosa26 ${ }^{\text {lacZ }}$ mice lines were maintained in a C57BL/6 background (Hayashi et al. 2003). All experimental procedures were performed with the approval of the animal research committee of Chongqing Medical University.

\section{PCR genotyping of transgenic mice}

Tbx18Cre mice were genotyped by PCR as previously described (Cai et al. 2008). Rosa26 $6^{\text {lacz }}$ (Soriano 1999) and Rosa26 ${ }^{\text {EYFP }}$ (Srinivas et al. 2001) mice lines were genotyped according to previously reported methods. Briefly, genomic DNA was extracted from mouse tail biopsies or yolk sacs by ethanol purification (Wang \& Storm 2006). For genotyping, a PCR Master Mix (Promega) was used with the following specific primers: Tbx18Cre mice, 5' GCC AGA GAA AGA GGA AAC GGC AAA $3^{\prime}$ and $5^{\prime}$ TCC CTG AAC ATG TCC ATC AGG TTC 3' (Cai et al. 2008); Rosa26 $6^{\text {lacZ }}, 5^{\prime}$ GCG AAG AGT TTG TCC TCA ACC 3' and 5' AAA GTC GCT CTG AGT TGT TAT 3' (Soriano 1999); Rosa26 ${ }^{\text {EYFP }}$, 5' AAG ACC GCG AAG AGT TTG TC $3^{\prime}$ and 5' AAA GTC GCT CTG AGT TGT TAT 3' (Srinivas et al. 2001). The PCR conditions are shown in Table 1.

\section{Detection of EYFP expression in whole-mount embryos and embryonic hearts}

EYFP reporter gene expression was determined using microdissected whole-mount embryos and embryonic hearts. The embryos and embryonic hearts were fixed in $4 \%$ paraformaldehyde (PFA) for $20 \mathrm{~min}$. The EYFP signal in whole-mount lateral views of embryos and embryonic hearts was observed immediately under a fluorescence stereomicroscope (Leica MZ 16 F, Wetzlar, Germany). After whole-embryo imaging, embryos and embryonic hearts were embedded within OCT and cryosectioned $(10 \mu \mathrm{m})$. EYFP fluorescence was observed in frozen tissue sections.

\section{X-gal staining}

We performed X-gal staining on whole-mount organs, embryos, and tissue sections (Nagy et al. 2007). Tbx18Cre/ Rosa2 $6^{\text {LacZ }}$ embryos and tissues were washed three times in PBS at room temperature to remove blood, fixed in cold $4 \%$ PFA at $4{ }^{\circ} \mathrm{C}$ for $90 \mathrm{~min}$, washed three times for $20 \mathrm{~min}$ each in $\beta$-gal washing buffer $\left(2 \mathrm{mmol} / \mathrm{l} \mathrm{MgCl}_{2}, 0.01 \%\right.$ sodium deoxycholate, $0.02 \%$ Nonidet-P40, and $100 \mathrm{mmol} / \mathrm{l}$ PBS buffer) at

Table 1 PCR conditions for genotyping.

\begin{tabular}{lcll}
\hline Step & Temperature $\left({ }^{\circ} \mathrm{C}\right)$ & Time & Note \\
\hline Tbx18Cre mice & & & \\
1 & 94 & $3 \mathrm{~min}$ & \\
2 & 94 & $30 \mathrm{~s}$ & \\
3 & 58 & $45 \mathrm{~s}$ & Repeat steps 2-4 \\
4 & 72 & $45 \mathrm{~s}$ & for 36 cycles \\
& & & \\
5 & 72 & $2 \mathrm{~min}$ & Hold \\
6 & 10 & & \\
Rosa26 $6^{\text {EYFP }}$ and & & & \\
Rosa26 & & & \\
1 & & $3 \mathrm{~min}$ & \\
2 & 94 & $30 \mathrm{~s}$ & \\
3 & 94 & $1 \mathrm{~min}$ & Repeat steps 2-4 \\
4 & 58 & $1 \mathrm{~min}$ & for 36 cycles \\
& 72 & & \\
5 & & & Hold \\
6 & 72 & & \\
\hline
\end{tabular}

www.reproduction-online.org 
room temperature, stained in X-gal staining solution $(5 \mathrm{mM}$ potassium ferrocyanide, $5 \mathrm{mM}$ potassium ferricyanide, $2 \mathrm{mmol} / \mathrm{LMgCl}_{2}, 0.01 \%$ sodium deoxycholate, $0.02 \% \mathrm{NP} 40$, $0.1 \% \mathrm{X}$-gal, and PBS buffer) for $16-24 \mathrm{~h}$ or longer at $37{ }^{\circ} \mathrm{C}$ in the dark, and washed three times at room temperature. The specimens were post-fixed in 4\% PFA for 1 week and then imaged with a stereomicroscope. They were then paraffinembedded and sectioned.

\section{Real time RT-PCR}

Total RNA from the testicles and ovaries of adult male and

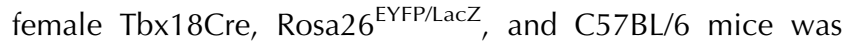
isolated using TRIzol Reagent (GIBCO). To assess the gene copy number of Cre and Tbx18 in each sample, cDNA was synthesized using the PrimeScript RT reagent kit Takara, Otsu, Japan; according to the manufacturer's instructions. Real-time qRT-PCR was performed with SYBR Green PCR master mix (Applied Biosystems) on a Real-Time PCR Detection System (Bio-Rad). GAPDH was used as a housekeeping gene for the normalization of the obtained results. The following primers were used: Cre (forward: 5'-ATG GCC CGC GCT GGA GTT TC $3^{\prime}$ and reverse: $5^{\prime}$-GCC ATC TTC CAG CAG GCG CA 3'), TBX18 (forward: 5'-CCG CAG GCC CCG AGA GTA GAT $3^{\prime}$ and reverse: $5^{\prime}$-AAC ATG CGC CTG CCG GCT T $3^{\prime}$ ), and GAPDH (forward: 5'-AAA TGG TGA AGG TCG GTG TGA AC $3^{\prime}$ and reverse: 5'-CAA CAA TCT CCA CTT TGC CAC TG 3').

\section{Immunofluorescence staining}

Immunofluorescence staining was performed as previously described (Cai et al. 2008). Testicular tissues from male mice and ovarian tissues from female mice were fixed in 4\% PFA for $\sim 4 \mathrm{~h}$, permeabilized in $0.5 \%$ Triton X-100 in PBS, and blocked in $5 \%$ BSA in PBS. Coverslips were incubated with goat antimouse Tbx18 antibody (Santa Cruz Biotechnology) at a 1:50 dilution in blocking buffer at $4{ }^{\circ} \mathrm{C}$ overnight. Samples were then washed and incubated with $\mathrm{Cy}_{3}$-labeled donkey anti-goat IgG secondary antibody (Beyotime, Shanghai, China) at a 1:100 dilution in blocking buffer for $1 \mathrm{~h}$ at $37^{\circ} \mathrm{C}$. Optical sections were visualized on a LEICA scanning laser confocal microscope.

\section{Statistics}

The gene copy numbers of Cre and Tbx18 mRNA are expressed as the mean \pm S.E.M. The significance of the differences between the two groups was evaluated by Student's two-tailed $t$-test. $P<0.05$ was considered significant.

\section{Results}

\section{Genetic characteristics of PM and RM lineage offspring}

We identified and screened mouse embryonic tissues by PCR to determine the Mendelian laws of inheritance in the PM and RM lineages (Fig. 1A, B, C, and D) (Kawamoto et al. 2000, Mao et al. 2001, Heinrich et al. 2004). The statistical analysis showed that approximately half of the offspring from the RM lineage inherited Cre and the Rosa26LacZ or EYFP reporter genes simultaneously (Table 2). The Cre-loxP-mediated Cre recombination frequency of the RM lineage double heterozygous mice was $\sim 1 / 2$, which does not correspond to a normal Mendelian distribution. However,

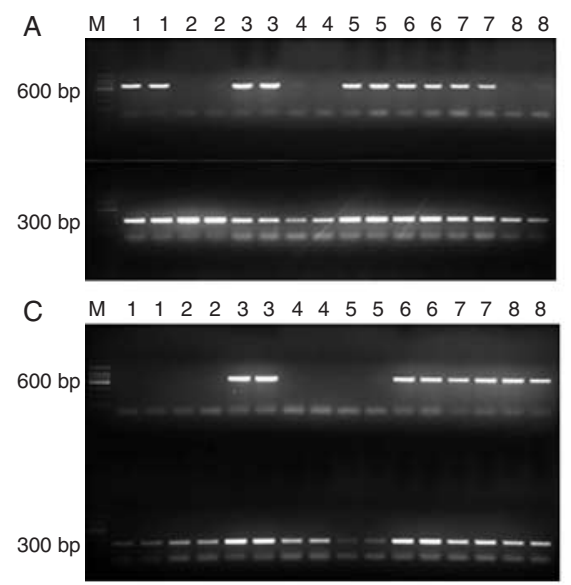

E

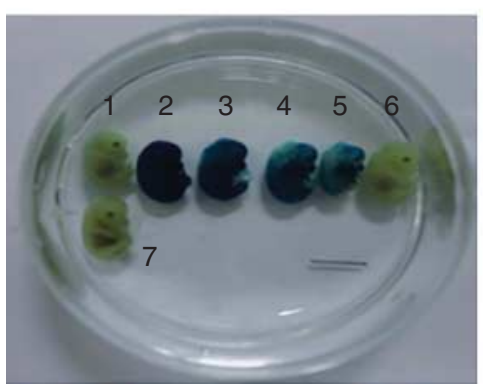

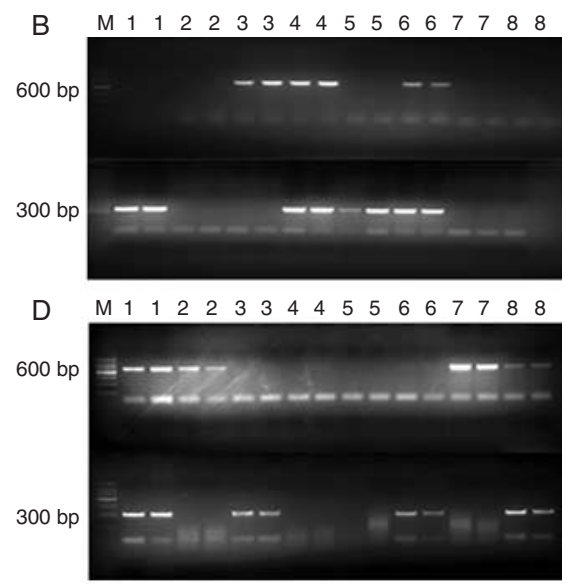

F

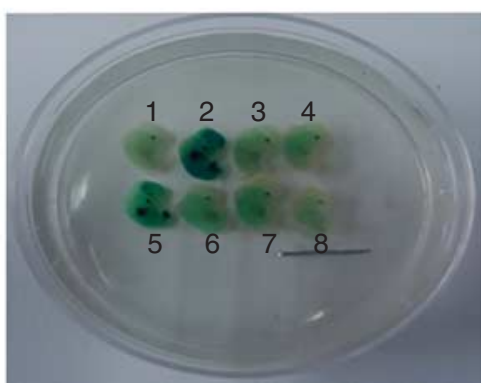

Figure 1 PCR genotyping results and LacZ expression in the offspring from PM and RM lineages. (A) PCR genotyping results from eight embryonic tissue samples obtained from the RM lineage: No. 1, 3, 5, 6, and 7 are double heterozygous Tbx18-Cre/Rosa26 $6^{\text {EYFP }}$ mice. (B) Genotypes of the eight embryonic tissues obtained from the PM lineage: No. 4 and 6 are double heterozygous Tbx18-Cre/ Rosa26 ${ }^{\text {EYFP }}$ mice. (C) RM lineage: No. 3, 6, 7, and 8 are double heterozygous Tbx18-Cre/ Rosa26 $6^{\text {LacZ }}$ mice. (D) PM lineage: No. 1 and 8 are double heterozygous Tbx18-Cre/ Rosa26 $6^{\text {LacZ }}$ mice. (E and F) LacZ reporter gene expression in the embryos of littermates. (E) RM lineage: No. 2, 3, 4, and 5 are double heterozygous embryos; the recombination frequency of Cre-mediated lacZ expression is 4/7. (F) PM lineage: No. 2 and 5 are double heterozygous embryos; the recombination frequency of Cre-mediated lac Z expression is $1 / 4$. 
Table 2 The double-heterozygous offspring of the PM and RM lineages and their Mendelian characteristics.

\begin{tabular}{|c|c|c|c|c|}
\hline & \multicolumn{2}{|c|}{ Tbx18Cre/Rosa26 ${ }^{\text {EYFP }}$} & \multicolumn{2}{|c|}{ Tbx18Cre/Rosa26 ${ }^{\text {LacZ }}$} \\
\hline & $\begin{array}{c}\text { PM } \\
\text { lineage }\end{array}$ & $\begin{array}{c}\text { RM } \\
\text { lineage }\end{array}$ & $\begin{array}{c}\text { PM } \\
\text { lineage }\end{array}$ & $\begin{array}{c}\mathrm{RM} \\
\text { lineage }\end{array}$ \\
\hline Total offspring ( $n$ ) & 126 & 114 & 88 & 101 \\
\hline $\begin{array}{l}\text { Double heterozy- } \\
\text { gous offspring }(n)\end{array}$ & 35 & 55 & 21 & 53 \\
\hline $\begin{array}{l}\text { Cre-loxP mediated } \\
\text { recombination } \\
\text { frequency }(\%)\end{array}$ & 27.8 & 48.2 & 23.9 & 52.5 \\
\hline
\end{tabular}

PM lineage: female Tbx18Cre mice crossed to male Rosa26R ${ }^{\text {lacz }}$ mice or female Tbx18Cre mice crossed to male Rosa26R ${ }^{\mathrm{EYFP}}$ mice.

This mating defined Cre as originating from maternal lineage models and was referred to as PM (positive mating). RM lineage: male Tbx18Cre mice crossed to female Rosa26R ${ }^{\text {lac Z }}$ mice or male Tbx $18 \mathrm{Cre}$ mice crossed to female Rosa26R ${ }^{\mathrm{EYFP}}$ mice. This mating defined Cre as originating from the paternal lineage model and was designated RM (reverse mating).

$\sim 1 / 4$ of the offspring from the PM lineage inherited both Cre and the Rosa26LacZ or EYFP reporter genes; this recombination frequency was in accordance with the Mendelian law (Table 2) (Hayashi et al. 2003, Cochrane et al. 2007). To reveal the genetic features of the PM and RM lineages, we detected LacZ expression in all of the littermate embryos. As shown in Fig. $1 \mathrm{E}$ and $\mathrm{F}$, the proportion of LacZ expression was 4/7 in all of the littermate embryos from the RM lineage. However, the recombination frequency of Cre-mediated lac $Z$ expression was $1 / 4$ in the PM lineage (Fig. 1F). These data indicated that the expression of reporter genes in fate-mapping mice of $\mathrm{Tbx} 18^{+}$progenitor cells from the RM lineage was not in accordance with Mendel's laws of inheritance.

\section{LacZ and EYFP reporter gene expression in PM and RM lineage offspring}

In the RM lineage, EYFP was detected in whole-mount embryos of Tbx18Cre/Rosa2 $6^{\text {LacZ }}$ mice at embryonic day 14.5 (E14.5). Similarly, intense blue staining was observed throughout the Tbx18Cre/Rosa26 $6^{\text {LacZ }}$ embryo (Fig. 2A and D), suggesting that Cre is expressed throughout the embryo. Indeed, the expression of EYFP and LacZ in RM lineage fate-mapping mice was nonspecific. However, EYFP expression was present in developing limb buds, hair follicles, and the hearts of Tbx18Cre/Rosa26 $6^{\text {EYFP }}$ embryos in the PM lineage at E14.5 (Fig. 2B). Similarly, LacZ was expressed in specific sites in the Tbx18Cre/Rosa26 $6^{\text {LacZ }}$ embryos of the PM lineage at E14.5, including the limb buds, heart, and hair follicles throughout the skin, which was identified by whole-mount X-Gal staining (Fig. 2E); this result confirmed those of previous studies (Kraus et al. 2001, Bussen et al. 2004, Cai et al. 2008, Trowe et al. 2008, Grisanti et al. 2013). The spatial and temporal specificity of the expression and localization of the LacZ reporter gene were consistent with EYFP in the PM lineage fatemapping models (Fig. 2B and E).

More in-depth observations were carried out at the tissue and organ levels with X-Gal staining and fluorescence analysis. In the PM lineage of Tbx18Cre/ Rosa26 $6^{\text {LacZ }}$ and Tbx18Cre/Rosa26 $6^{\text {EYFP }}$ mice, the LacZ and EYFP reporter genes were expressed only at specific sites in the embryonic heart, including the coronary vasculature, epicardium, and part of the heart tissue (Fig. 3B, E, H, and K), as previously noted (Cai et al. 2008, Wu et al. 2013). In contrast, LacZ and EYFP were detected in the myocardium of whole-mount hearts in the RM lineages (Fig. 3A, D, G, and J), which suggested that the $\mathrm{Tb} 18^{+}$cell fate-mapping models from RM lineages drive Cre recombinase for excessive recombination of floxed alleles in the developing hearts of embryos. The distinct expression pattern indicated that the reporter genes did not accurately and reliably trace the cell differentiation in fate-mapping models of paternal Cre.

\section{Expression of Tbx18 and Cre in the germline}

The reason for the aforementioned infidelity in the RM lineages is unclear. To determine why reporter genes did not accurately and reliably trace cell differentiation, we measured Tbx18 and Cre expression in the germline. Real-time PCR showed that Cre mRNA was only expressed in the testicles of male Tbx18Cre mice (Fig. 4A). Additionally, Tbx18 mRNA was expressed in
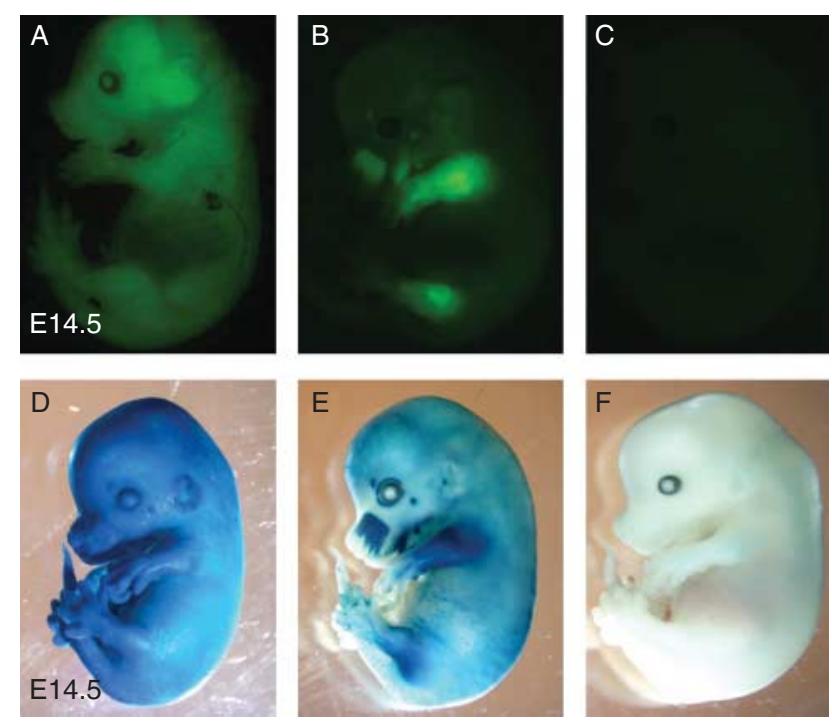

Figure 2 EYFP and LacZ reporter gene expression in whole-mount embryos (E14.5). (A) EYFP expression was detected in whole-mount embryos of the RM lineage at E14.5. (B) EYFP reporter gene expression was restricted to specific places in the embryo from the PM lineage. (C) EYFP was not expressed in the embryos of the WT C57 mice at E14.5. (D) LacZ gene expression was detected throughout the wholemount embryo in the RM lineage. (E) LacZ reporter gene expression was restricted to specific places in the embryo from the PM lineage. (F) LacZ was not expressed in the embryos of WT C57 mice at E14.5. 

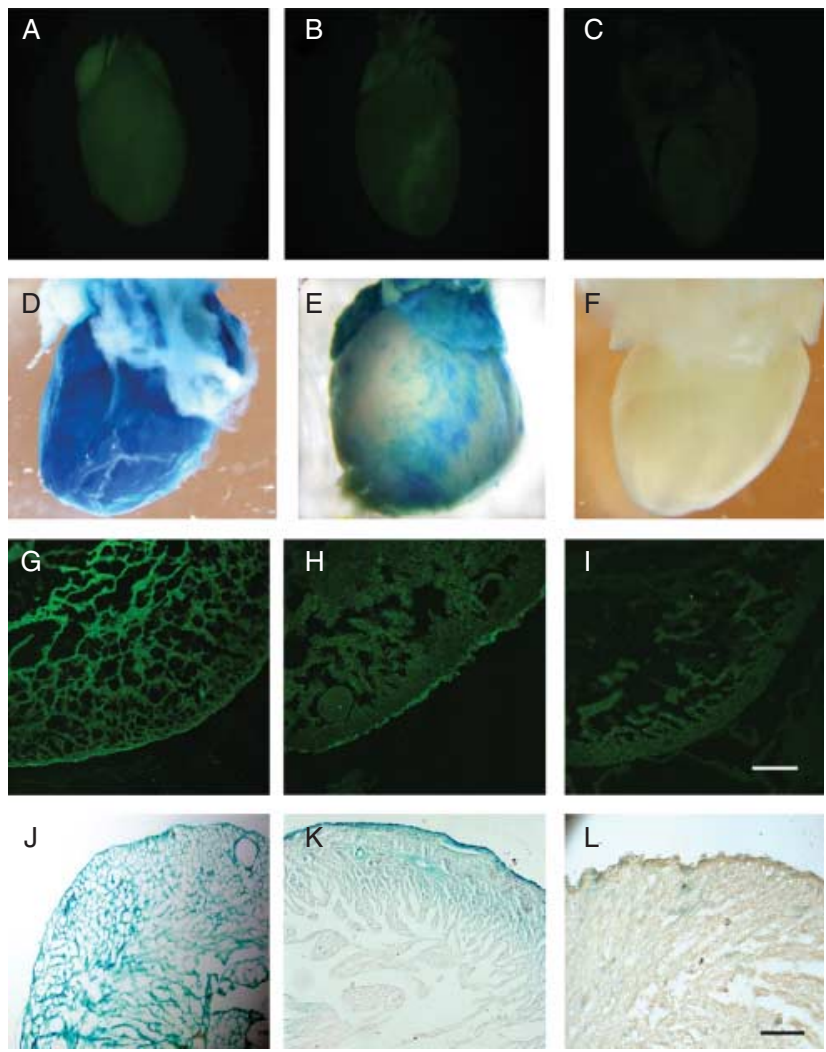

Figure 3 EYFP and LacZ reporter gene expression in whole-mount hearts and sections of heart tissues. (A and D) EYFP and LacZ were detected throughout the whole-mount embryonic heart in the RM lineage at E14.5. (B and E) EYFP and LacZ were restricted to specific places in the heart in the PM lineage. (C and F) EYFP and LacZ were not expressed in the heart of the WT C57 mice at E14.5. (G and J) EYFP and LacZ were expressed throughout the cardiac sections in the RM lineage. ( $\mathrm{H}$ and $\mathrm{K}$ ) EYFP and LacZ were expressed only in specific places in the cardiac sections from the PM lineage. (I and L) EYFP and LacZ were not expressed in the cardiac sections of WT C57 mice. Scale bar $=150 \mu \mathrm{m}$.

the testicles of male Tbx18Cre, Rosa $26^{\text {LacZ, }}$ and Rosa26 ${ }^{\text {EYFP }}$ mice, but almost no expression was detected in the ovaries of female mice (Fig. 4A, B, and C). Immunofluorescence assays revealed that Tbx18 protein was only expressed in the testicles of male Tbx18Cre, Rosa26 $6^{\text {LacZ }}$, Rosa26 $6^{\text {EYFP }}$, and WT C57 mice, and almost no expression was detected in the ovaries of female mice (Fig. 5A, B, C, D, E, F, G, and H). Consequently, in the RM lineage with paternal Cre origin, Tbx18 mRNA and protein were expressed in sperm cells, which may explain this infidelity.

\section{Expression of reporter genes in the germline}

As shown in Fig. 2B and E, the reporter genes EYFP and LacZ can accurately and reliably trace cell differentiation in the PM lineage of Tbx 18 Cre/Rosa $26^{\text {LacZ }}$ and Tbx18Cre/Rosa26 EYFP mice. In addition, Tbx18CreRosa26 double heterozygous mice can be used to trace the expression of endogenous Tbx18. We detected the expression of EYFP and Lacz reporter genes in the germline. Whole-mount X-gal staining revealed Lacz expression in the testicles of male Tbx18Cre-Rosa26 double heterozygous mice (Fig. 6A). Furthermore, X-gal staining on cryosections showed Lacz expression within the seminiferous tubule and sperm cells of testicles (Fig. 6B and C). Similarly, immunofluorescence analysis showed EYFP expression within the seminiferous tubule and sperm cells of testicles (Fig. 6E and F). EYFP and Tbx18 were coexpressed in the seminiferous tubules and sperm cells (Fig. 6H), but X-gal staining did not detect Lacz in cryosections of the ovarian follicle (Fig. 6D). These results confirmed that Cre-loxP-mediated prerecombination in zygotes is due to the early activation of Cre expression in the testicles when Tbx18Cre is transmitted paternally.

\section{Discussion}

Genetic lineage tracing techniques, which are based on the Cre-loxP system, have been used extensively in developmental biology to reveal the origin of progenitor cells in the early embryo (Kretzschmar \& Watt 2012, Laforest et al. 2014). The expression of Cre recombinase is under the control of cell or tissue-specific promoters in Cre mouse lines. A Cre mouse is crossed with a Cre reporter mouse, such as Rosa26lacZ or Rosa26EYFP, which contains the EYFP or LacZ reporter genes flanked by a loxP-STOP-loxP sequence (Srinivas et al. 2001, Muzumdar et al. 2007, Kretzschmar \& Watt 2012,
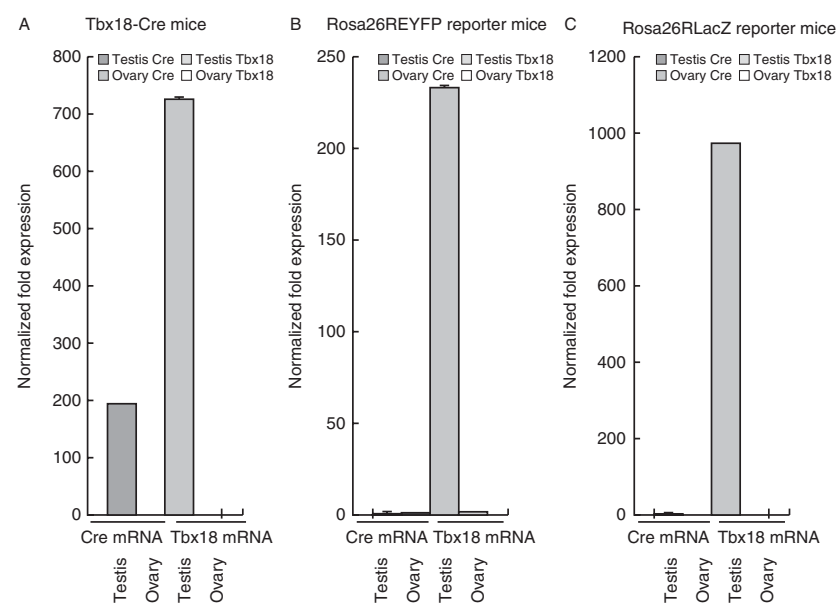

Figure 4 The expression of Cre recombinase and Tbx18 mRNA in the mouse reproductive system was detected by real-time qRT-PCR. (A) Cre and Tbx18 mRNA were expressed in testes of male heterozygous Tbx18-Cre mice but not in ovarian cells. (B) Tbx18 mRNA, but not Cre mRNA, was expressed in the testes of male heterozygous Rosa26R ${ }^{\text {EYFP }}$ reporter mice, though neither Tbx18 nor Cre was expressed in the ovaries of female heterozygous Rosa26 ${ }^{\mathrm{EYFP}}$ reporter mice. (C) Tbx18 mRNA, but not Cre mRNA, was expressed in the testes of male heterozygous Rosa26R ${ }^{\text {LacZ }}$ reporter mice; neither Tbx18 nor Cre was expressed in the, ovaries. 

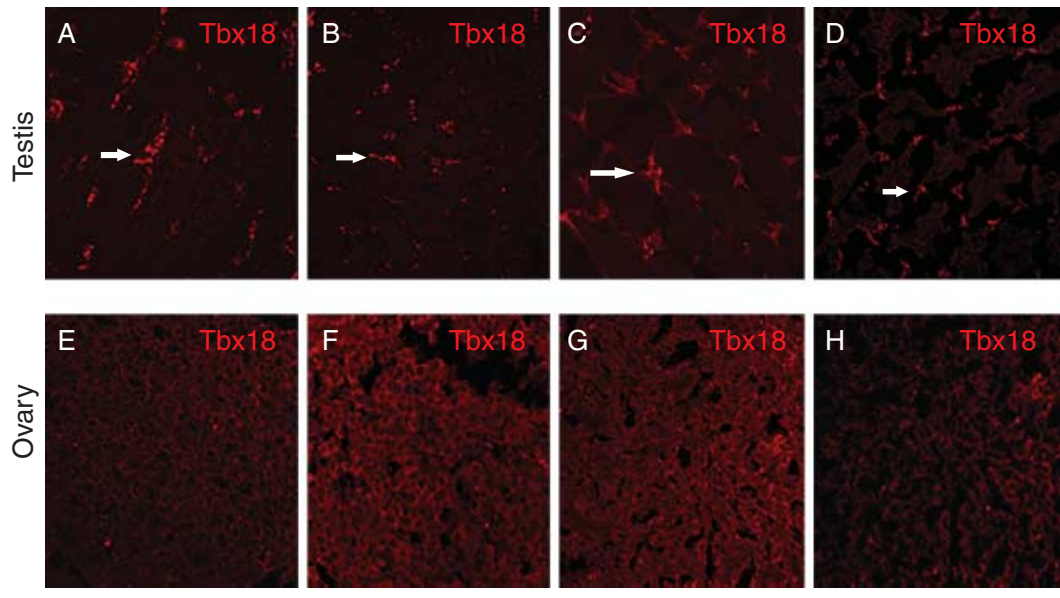

Tbx18Cre mice
Rosa26 EYFP mice

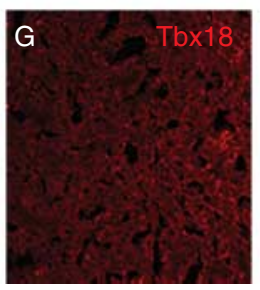

Rosa26 Lacz mice

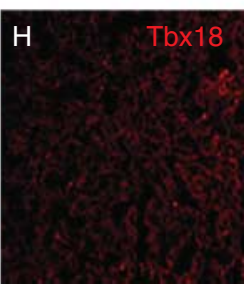

C57 mice
Figure 5 Immunofluorescent analysis of Tbx18 protein expression in frozen sections of testicles $(\mathrm{A}, \mathrm{B}, \mathrm{C}$, and $\mathrm{D})$ and ovaries $(\mathrm{E}, \mathrm{F}, \mathrm{G}$, and $\mathrm{H})$ in Tbx18cre, Rosa26EYFP, Rosa26Lacz, and C57BL/6 mice. (A, B, C, and D) Tbx18 protein (red, Cy3) was detected in testicular tissue, especially Leydig cells (marked with white arrows) of male Tbx18-Cre, Rosa26EYFP, Rosa26Lacz, and WT C57BL/6 mice. $(\mathrm{E}, \mathrm{F}, \mathrm{G}$, and $\mathrm{H})$ Tbx18 protein was not detected in ovaries of female Tbx18-Cre, Rosa26EYFP, Rosa26Lacz, or C57BL/6 mice.
Blanpain \& Simons 2013, Kraus et al. 2014). In animals expressing both Cre and a floxed reporter gene, Cre activates EYFP or LacZ reporter gene expression by excising the STOP sequence specifically in the traced cells (Kretzschmar \& Watt 2012). However, nonspecific reporter gene expression may occur in fate-mapping mice. The nonspecific expression pattern of reporter genes is caused by various factors, such as the differences in Cre lines and genes, Cre-mediated chromosome loss, death of proliferating cells, cytotoxic effects, or epigenetic modifications (Lomeli et al. 2000, Silver \& Livingston 2001, Ramirez et al. 2004, Rassoulzadegan et al. 2006, Cochrane et al. 2007, Lee et al. 2013). However, it is not clear whether reporter genes are influenced by the origins of Cre from the paternal or maternal lineage in genetic lineage tracing mice. In the current study, we have compared the difference in reporter gene expression between paternal and maternal Cre lineages.
The Tbx18Cre/R26LacZ and Tbx18Cre/R26EYFP double heterozygous mice were produced for tracing the fate of $\mathrm{Tbx}^{+} 8^{+}$progenitor cells. Genetic fate mapping revealed that $\mathrm{Tbx}^{+} 8^{+}$progenitor cells play a critical role in the development of the kidneys, urinary tract, heart, limbs, somites, and hair follicles in mice (Airik et al. 2006, Cai et al. 2008, Trowe et al. 2008, Christoffels et al. 2009, Grisanti et al. 2013, Wu et al. 2013, Xu et al. 2014). Interestingly, we found that the LacZ and EYFP reporter genes were expressed in the whole mouse embryo and whole-mount hearts. Therefore, LacZ and EYFP did not accurately and reliably trace cell differentiation in fate-mapping models. Nevertheless, the reason for the nonspecific expression of EYFP and LacZ in the RM lineage is unclear. As shown in Figs 4, 5, and 6, Tbx 18 mRNA and protein were expressed in sperm cells, but no expression was detected in the ovaries of female Cre mice. This result suggests that some sperm cells express Tbx18 if the zygote has Tbx18Cre
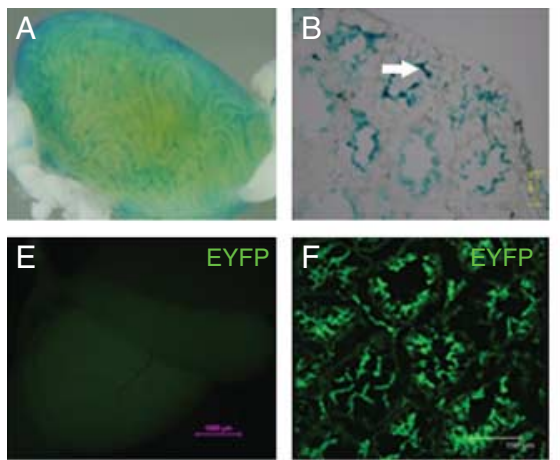
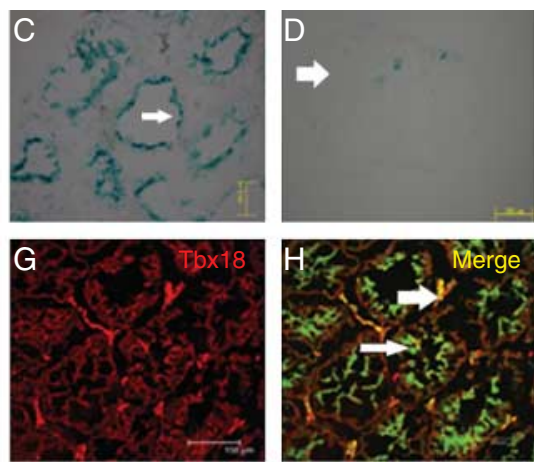

Figure $6 \mathrm{LacZ}$ and EYFP expression in the testicles and ovaries of Tbx18Cre-Rosa26 $6^{\text {Lacz }}$ and Tbx18Cre-Rosa26 ${ }^{\text {EYFP }}$ double heterozygous mice from the PM lineage. (A, B, C, and D) X-gal staining in testicle tissue. (A) X-gal staining on whole-mount testicle shows LacZ expression. (B, and C) X-gal staining on cryosections shows LacZ expression within the seminiferous tubule and sperm cells (marked with white arrows). (D) X-gal staining on cryosections shows a lack of LacZ expression within the ovarian follicle of the ovary (marked with white arrows). (E, F, G, and H) EYFP expression in testicle tissue. (E) EYFP protein (green) is shown in whole-mount images of testicle tissue. ( $F$ and $G$ ) Immunofluorescent analysis shows EYFP expression within the seminiferous tubule and sperm cells of testicle tissue; EYFP and Tbx18 are coexpressed (marked with white arrows). 
and a Rosa indicator allele simultaneously, leading to excision in zygote cells. In the embryonic tissue from these zygotes, the embryos receiving the flanked indicator allele will show reporter activity in every single cell. Hence, in the RM lineage-derived double heterozygous Tbx18Cre/Rosa26 $6^{\text {LacZ }}$ and Tbx18Cre/Rosa26 $6^{\text {EYFP }}$ zygotes, Cre expression (activated by the Tbx18 promoter) and loxP-flanked allele expression (from the Rosa26 reporter mouse) appeared simultaneously. Thus, Cre-loxP recombination occurs early in zygotes or all embryonic stem cells when Tbx 18 Cre is transmitted paternally, which induced whole-mount expression of the reporter gene in all of the embryonic tissues. Based on our results, we conclude that Cre-mediated specific recombination in fate-mapping models of spermexpressed transcription factors is influenced by the paternal origin of Cre.

A successful and specific fate-mapping model is important for research on stem cells and progenitor cells and in the medical field. The Cre-loxP system is an essential tool for studying gene function, allowing genetic inactivation or endogenous activation in a temporally or spatially regulated manner. However, this system also has caveats that affect loxP sequence recombination, such as nonspecific expression patterns, Cre toxicity, and a variation of Cre recombination efficiency, all of which may affect phenotypic interpretation (Schmidt-Supprian \& Rajewsky 2007). Our results showed that sperm-expressed genes might be influenced by the paternal origin of Cre. Thus, when Cre-mediated recombination in the paternal germline is applied to a fate-mapping model of sperm-expressed genes, the expression of reporter genes in the reproductive system may be nonspecific (Wagner et al. 2001, Hafner et al. 2004, Ramirez et al. 2004, Casola et al. 2006). Our study mainly discussed Cre-mediated recombination in the reproductive system and demonstrated that different mating schemes arising from either paternally or maternally inherited transgenes may lead to different experimental outcomes. Because Cre-LoxP recombination can take place early in the zygote stage in an established fate-mapping Cre line, specific genes or transcription factors that are expressed in the reproductive system may lead to the expression of the reporter gene and support incorrect conclusions about cell differentiation.

Taken together, our results indicate that Cre-loxP recombination occurs early in zygotes or all embryonic stem cells when Tbx18Cre is transmitted paternally; the resultant nonspecific expression patterns induce reporter gene expression in all of the embryonic tissues. Consequently, Cre-mediated specific recombination in fate-mapping models of sperm-expressed genes or transcription factors may be influenced by the paternal origin of Cre. In our study, efficient and specific recombination occurred in the embryo when the Cre transgene was inherited from maternal lines; therefore, mating by maternal inheritance may be a feasible way to address the potential influences of sperm-expressed genes in fate-mapping models. In conclusion, a careful experimental design is warranted when using the CreloxP system to trace spatial, temporal, or tissue-specific gene fate mapping.

\section{Declaration of interest}

The authors declare that there is no conflict of interest that could be perceived as prejudicing the impartiality of the research reported.

\section{Funding}

This work was supported by grants from the National Natural Science Foundation of China (NSFC, No. 30971213, 81270211, 81100088), Higher Specialized Research Fund for the Doctoral Program (20125503110009), and Science and Technology Research Projects of Chongqing Education Commission (KJ130324).

\section{Acknowledgements}

The authors thank S M Evans (University of California, San Diego, CA, USA) for providing Tbx18Cre mice.

\section{References}

Airik R, Bussen M, Singh MK, Petry M \& Kispert A 2006 Tbx18 regulates the development of the ureteral mesenchyme. Journal of Clinical Investigation 116 663-674. (doi:10.1172/JCl26027)

Blanpain C \& Simons BD 2013 Unravelling stem cell dynamics by lineage tracing. Nature Reviews. Molecular Cell Biology 14 489-502. (doi:10.1038/nrm3625)

Bohnenpoll T, Bettenhausen E, Weiss AC, Foik AB, Trowe MO, Blank P, Airik R \& Kispert A 2013 Tbx18 expression demarcates multipotent precursor populations in the developing urogenital system but is exclusively required within the ureteric mesenchymal lineage to suppress a renal stromal fate. Developmental Biology 380 25-36. (doi:10.1016/j.ydbio.2013.04.036)

Bussen M, Petry M, Schuster-Gossler K, Leitges M, Gossler A \& Kispert A 2004 The T-box transcription factor Tbx18 maintains the separation of anterior and posterior somite compartments. Genes \& Development 18 1209-1221. (doi:10.1101/gad.300104)

Cai CL, Martin JC, Sun Y, Cui L, Wang L, Ouyang K, Yang L, Bu L, Liang X, Zhang X et al. 2008 A myocardial lineage derives from Tbx18 epicardial cells. Nature 454 104-108. (doi:10.1038/nature06969)

Casola S, Cattoretti G, Uyttersprot N, Koralov SB, Seagal J, Hao Z, Waisman A, Egert A, Ghitza D \& Rajewsky K 2006 Tracking germinal center B cells expressing germ-line immunoglobulin $\gamma 1$ transcripts by conditional gene targeting. PNAS 103 7396-7401. (doi:10.1073/pnas. 0602353103)

Christoffels VM, Grieskamp T, Norden J, Mommersteeg MT, Rudat C \& Kispert A 2009 Tbx18 and the fate of epicardial progenitors. Nature 458 E8-E9; discussion E9-10. (doi:10.1038/nature07916)

Cochrane RL, Clark SH, Harris A \& Kream BE 2007 Rearrangement of a conditional allele regardless of inheritance of a Cre recombinase transgene. Genesis 45 17-20. (doi:10.1002/dvg.20259)

Grisanti L, Clavel C, Cai X, Rezza A, Tsai SY, Sennett R, Mumau M, Cai CL \& Rendl M 2013 Tbx18 targets dermal condensates for labeling, isolation, and gene ablation during embryonic hair follicle formation. Journal of Investigative Dermatology 133 344-353. (doi:10. 1038/jid.2012.329) 
Hafner M, Wenk J, Nenci A, Pasparakis M, Scharffetter-Kochanek K, Smyth N, Peters T, Kess D, Holtkotter O, Shephard P et al. 2004 Keratin 14 Cre transgenic mice authenticate keratin 14 as an oocyte-expressed protein. Genesis 38 176-181. (doi:10.1002/gene.20016)

Hayashi S, Tenzen T \& McMahon AP 2003 Maternal inheritance of Cre activity in a Sox2Cre deleter strain. Genesis 37 51-53. (doi:10.1002/gene.10225)

Heinrich AC, Pelanda R \& Klingmuller U 2004 A mouse model for visualization and conditional mutations in the erythroid lineage. Blood 104 659-666. (doi:10.1182/blood-2003-05-1442)

Kawamoto S, Niwa H, Tashiro F, Sano S, Kondoh G, Takeda J, Tabayashi K \& Miyazaki J 2000 A novel reporter mouse strain that expresses enhanced green fluorescent protein upon Cre-mediated recombination. FEBS Letter 470 263-268. (doi:10.1016/S0014-5793(00)01338-7)

Kraus F, Haenig B \& Kispert A 2001 Cloning and expression analysis of the mouse T-box gene Tbx18. Mechanisms of Development 100 83-86. (doi:10.1016/S0925-4773(00)00494-9)

Kraus P, Sivakamasundari V, Xing X \& Lufkin T 2014 Generating mouse lines for lineage tracing and knockout studies. Methods in Molecular Biology 1194 37-62.

Kretzschmar K \& Watt FM 2012 Lineage tracing. Cell 148 33-45. (doi:10.1016/j.cell.2012.01.002)

Laforest B, Bertrand N \& Zaffran S 2014 Genetic lineage tracing analysis of anterior Hox expressing cells. Methods in Molecular Biology 1196 $37-48$.

Lee KY, Russell SJ, Ussar S, Boucher J, Vernochet C, Mori MA, Smyth G, Rourk M, Cederquist C, Rosen ED et al. 2013 Lessons on conditional gene targeting in mouse adipose tissue. Diabetes 62 864-874. (doi:10.2337/db12-1089)

Liang X, Wang G, Lin L, Lowe J, Zhang Q, Bu L, Chen Y, Chen J, Sun Y \& Evans SM 2013 HCN4 dynamically marks the first heart field and conduction system precursors. Circulation Research 113 399-407. (doi:10.1161/CIRCRESAHA.113.301588)

Lomeli H, Ramos-Mejia V, Gertsenstein M, Lobe CG \& Nagy A 2000 Targeted insertion of Cre recombinase into the TNAP gene: excision in primordial germ cells. Genesis 26 116-117. (doi:10.1002/(SICI)1526968X(200002)26:2<116::AID-GENE4 > 3.0.CO;2-X)

Mao X, Fujiwara Y, Chapdelaine A, Yang H \& Orkin SH 2001 Activation of EGFP expression by Cre-mediated excision in a new ROSA26 reporter mouse strain. Blood 97 324-326. (doi:10.1182/blood.V97.1.324)

Muzumdar MD, Tasic B, Miyamichi K, Li L \& Luo L 2007 A global doublefluorescent Cre reporter mouse. Genesis 45 593-605. (doi:10.1002/dvg. 20335)

Nagy A, Gertsenstein M, Vintersten K \& Behringer R 2007 Staining whole mouse embryos for $\beta$-galactosidase (lacZ) activity. $\mathrm{CSH}$ protocols $\mathbf{2 0 0 7}$ pdb.prot4725. (doi:10.1101/pdb.prot472)

Ramirez A, Page A, Gandarillas A, Zanet J, Pibre S, Vidal M, Tusell L, Genesca A, Whitaker DA, Melton DW et al. 2004 A keratin K5Cre transgenic line appropriate for tissue-specific or generalized Cremediated recombination. Genesis 39 52-57. (doi:10.1002/gene.20025)

Rassoulzadegan M, Grandjean V, Gounon P, Vincent S, Gillot I \& Cuzin F 2006 RNA-mediated non-mendelian inheritance of an epigenetic change in the mouse. Nature 441 469-474. (doi:10.1038/nature04674)
Schmidt-Supprian M \& Rajewsky K 2007 Vagaries of conditional gene targeting. Nature Immunology 8 665-668. (doi:10.1038/ni0707-665)

Silver DP \& Livingston DM 2001 Self-excising retroviral vectors encoding the Cre recombinase overcome Cre-mediated cellular toxicity. Molecular Cell 8 233-243. (doi:10.1016/S1097-2765(01)00295-7)

Smith L 2011 Good planning and serendipity: exploiting the Cre/Lox system in the testis. Reproduction 141 151-161. (doi:10.1530/REP-10-0404)

Soriano P 1999 Generalized lacZ expression with the ROSA26 Cre reporter strain. Nature Genetics 21 70-71. (doi:10.1038/5007)

Srinivas S, Watanabe T, Lin CS, William CM, Tanabe $Y$, Jessell TM \& Costantini F 2001 Cre reporter strains produced by targeted insertion of EYFP and ECFP into the ROSA26 locus. BMC Developmental Biology 1 4. (doi:10.1186/1471-213X-1-4)

Trowe MO, Maier H, Schweizer M \& Kispert A 2008 Deafness in mice lacking the T-box transcription factor Tbx18 in otic fibrocytes. Development 135 1725-1734. (doi:10.1242/dev.014043)

Wagner KU, McAllister K, Ward T, Davis B, Wiseman R \& Hennighausen L 2001 Spatial and temporal expression of the Cre gene under the control of the MMTV-LTR in different lines of transgenic mice. Transgenic Research 10 545-553. (doi:10.1023/A:1013063514007)

Wang Z \& Storm DR 2006 Extraction of DNA from mouse tails. Biotechniques 41 410, 412. (doi:10.2144/000112255)

Wang Y, Tripathi P, Guo Q, Coussens M, Ma L \& Chen F 2009 Cre/lox recombination in the lower urinary tract. Genesis 47 409-413. (doi:10.1002/dvg.20515)

Wehn AK \& Chapman DL 2010 Tbx18 and Tbx15 null-like phenotypes in mouse embryos expressing Tbx6 in somitic and lateral plate mesoderm. Developmental Biology 347 404-413. (doi:10.1016/j.ydbio.2010.09.001)

Wiese C, Grieskamp T, Airik R, Mommersteeg MT, Gardiwal A, de Gier-de Vries C, Schuster-Gossler K, Moorman AF, Kispert A \& Christoffels VM 2009 Formation of the sinus node head and differentiation of sinus node myocardium are independently regulated by Tbx 18 and Tbx3. Circulation Research 104 388-397. (doi:10.1161/CIRCRESAHA.108. 187062)

Wu SP, Dong XR, Regan JN, Su C \& Majesky MW 2013 Tbx18 regulates development of the epicardium and coronary vessels. Developmental Biology 383 307-320. (doi:10.1016/j.ydbio.2013.08.019)

Xu J, Nie X, Cai X, Cai CL \& Xu PX 2014 Tbx18 is essential for normal development of vasculature network and glomerular mesangium in the mammalian kidney. Developmental Biology 391 17-31. (doi:10.1016/ j.ydbio.2014.04.006)

Yan J, Zhang L, Xu J, Sultana N, Hu J, Cai X, Li J, Xu PX \& Cai CL 2014 Smad4 regulates ureteral smooth muscle cell differentiation during mouse embryogenesis. PLOS ONE 9 e104503. (doi:10.1371/journal. pone.0104503)

Received 13 January 2015

First decision 9 February 2015

Revised manuscript received 17 June 2015

Accepted 7 July 2015 